\title{
The incidence of training responsiveness to cardiorespiratory fitness and cardiometabolic measurements following individualized and standardized exercise prescription: study protocol for a randomized controlled trial
}

Ryan M. Weatherwax ${ }^{1,3^{*}}$, Nigel K. Harris ${ }^{1}$, Andrew E. Kilding ${ }^{2}$ and Lance C. Dalleck ${ }^{3}$

\begin{abstract}
Background: There is individual variability to cardiorespiratory fitness (CRF) training, but the underlying cause is not well understood. Traditionally, a standardized approach to exercise prescription has utilized relative percentages of maximal heart rate, heart rate reserve ( $\mathrm{HRR})$, maximal oxygen uptake $\left(\mathrm{VO}_{2} \mathrm{max}\right)$, or $\mathrm{VO}_{2}$ reserve to establish exercise intensity. However, this model fails to take into consideration individual metabolic responses to exercise and may attribute to the variability in training responses. It has been proposed that an individualized approach would take into consideration metabolic responses to exercises to increase responsiveness to training.

Methods: In this randomized control trial, participants will undergo a 12-week exercise intervention using individualized (ventilatory thresholds) and standardized (HRR) methods to prescribe CRF training intensity. Following the intervention, participants will be categorized as responders or non-responders based on changes in maximal aerobic abilities. Participants who are non-responders will complete a second 12-week intervention in a crossover design to determine whether they can become responders with a differing exercise prescription. There are four main research outcomes: (1) determine the cohort-specific technical error to use in the categorization of response rate; (2) determine if an individualized intensity prescription is superior to a standard approach in regards to $\mathrm{VO}_{2}$ max and cardiometabolic risk factors; (3) investigate the time course changes throughout 12 weeks of CRF training between the two intervention groups; and (4) determine if non-responders can become responders if the exercise prescription is modified.
\end{abstract}

Discussion: The findings from this research will provide evidence on the effectiveness of individualized exercise prescription related to training responsiveness of $\mathrm{VO}_{2}$ max and cardiometabolic risk factors compared to a standardized approach and further our understanding of individual exercise responses. If the individualized approach proposed is deemed effective, it may change the way exercise specialists prescribe exercise intensity to enhance training responsiveness.

Trial registration: ClinicalTrials.gov, NCT02868710. Registered on 15 August 2016.

Keywords: Responder, Non-responder, Exercise prescription, Ventilatory threshold, Cardiovascular disease, Primary prevention

\footnotetext{
* Correspondence: rweatherwax@western.edu

${ }^{1}$ Auckland University of Technology, Human Potential Centre, Auckland, New

Zealand

${ }^{3}$ Department of Recreation, Exercise, and Sport Science, Western State

Colorado University, Gunnison, CO, USA

Full list of author information is available at the end of the article
} 


\section{Background}

Heterogeneity in the response to exercise training first received attention in the 1980s [1] with a series of standardized studies investigating trainability of sedentary adults. Among these studies was an investigation into responses of maximal aerobic power in which it was reported that interindividual differences ranged from 5\% to $88 \%$ [2]. Even though these original findings were reported over 30 years ago, substantial individual variability in response to prescribed exercise regimes remains a poorly understood phenomena. Nonetheless, it has been purported that a more individualized approach to the exercise prescription may enhance training efficacy and limit training unresponsiveness. For instance, it has been acknowledged as far back as the late 1970 s that utilizing a relative percent method (i.e., \% heart rate reserve [HRR]) to establish exercise intensity fails to account for individual metabolic responses to exercise [3]. Nevertheless, the relative percent concept remains the gold standard recommendation for exercise intensity [4]. It is both plausible and practical to think that an intensity set based on an individual's threshold measurement (i.e. ventilatory threshold) will not only encourage more positive physiological adaptations, but may account for some of the variability in training responsiveness by taking into consideration individual metabolic differences. Based on an extensive search of the literature, to our knowledge, there is only one investigation that set out to determine the incidence of response based on exercise prescription using standard methods (\%HRR) compared to individualized methods (threshold based) in which they found $100 \%$ of the individualized group responded in a positive manner [5]. However, this investigation had several limitations including a modest intervention duration, only reported maximal oxygen uptake $\left(\mathrm{VO}_{2} \mathrm{max}\right)$ changes, and sourced measurements for biological variability to use as criteria for response rate rather than testing for biological variability within the laboratory where data were collected.

A notable factor that confounds current understanding of training response variability is the absence of a set definition in the literature of how to interpret a response (i.e. what classifies someone as a responder or nonresponder). Indeed, criteria to determine incidence of response for changes in $\mathrm{VO}_{2}$ max have included classifying a fixed proportion of the lowest training response [6], absolute changes in pre- to post-intervention values $[7,8]$, and a change of more than one standard deviation [9]. More recently, it has been proposed that technical error (TE), the combination of day-to-day biological variability and measurement error, should be applied to categorize response rate [10]. If these values are considered for each research cohort to report incidence of response, there would be greater consistency of reporting results within the literature to provide further insight on individual variability. Moreover, interpretation of the individual variability in training responsiveness is limited due in part to the standard practice of past studies only reporting group means and standard deviations. With reporting of only the mean and standard deviation, results of the intervention may not be applicable to all since there is a lack of understanding related to the individual variability of the investigation.

This trial will be the first investigation to address the incidence of response of $\mathrm{VO}_{2}$ max and cardiometabolic risk factors following individualized and standardized cardiorespiratory fitness (CRF) training using a specific cohortcalculated TE as the criteria for response. Similarly, this will be the first study to evaluate the efficacy of modifying the CRF training intensity prescription for non-responsive participants (based on changes of $\mathrm{VO}_{2} \mathrm{max}$ ) to investigate subsequent training responsiveness.

\section{Research aims}

The objective of this research is to determine the incidence of response to $\mathrm{VO}_{2} \max$ after implementation of a standardized (\%HRR) and individualized (based on first ventilatory threshold [VT1] and second ventilatory threshold [VT2]) approach to exercise prescription in a community wellness program for 12 weeks. The primary measurement outcome will be $\mathrm{VO}_{2}$ max with secondary outcomes of total cholesterol (TC), high-density lipoprotein (HDL), low-density lipoprotein (LDL), triglycerides, blood glucose, and resting heart rate (HR) and blood pressure (BP). Thus, the main research aim is to determine whether an individualized exercise prescription decreases the incidence of non-response to CRF and cardiometabolic measurements compared to the standardized approach. Similarly, another primary research aim is to determine if changing the prescription of CRF intensity for a subsequent 12-week intervention elicits more responsiveness in previously categorized nonresponders (based on $\mathrm{VO}_{2} \max$ responses). A key secondary aim of this research is to establish whether there are differences in the time course changes of $\mathrm{VO}_{2} \max$ and cardiometabolic risk factors between the experimental groups every fourth week during the 12-week intervention.

\section{Methods/Design}

The Standard Protocol Items: Recommendations for Interventional Trials (SPIRIT) guidelines [11] have been taken into consideration for the planning of this trial (Additional file 1). The overall study (Fig. 1) is a randomized control trial with participants completing a CRF training study 3 days a week for a duration of 12 weeks using a standardized (\%HRR) and an individualized (based on VT1 and VT2) approach (see Additional file 2: overall SPIRIT study 


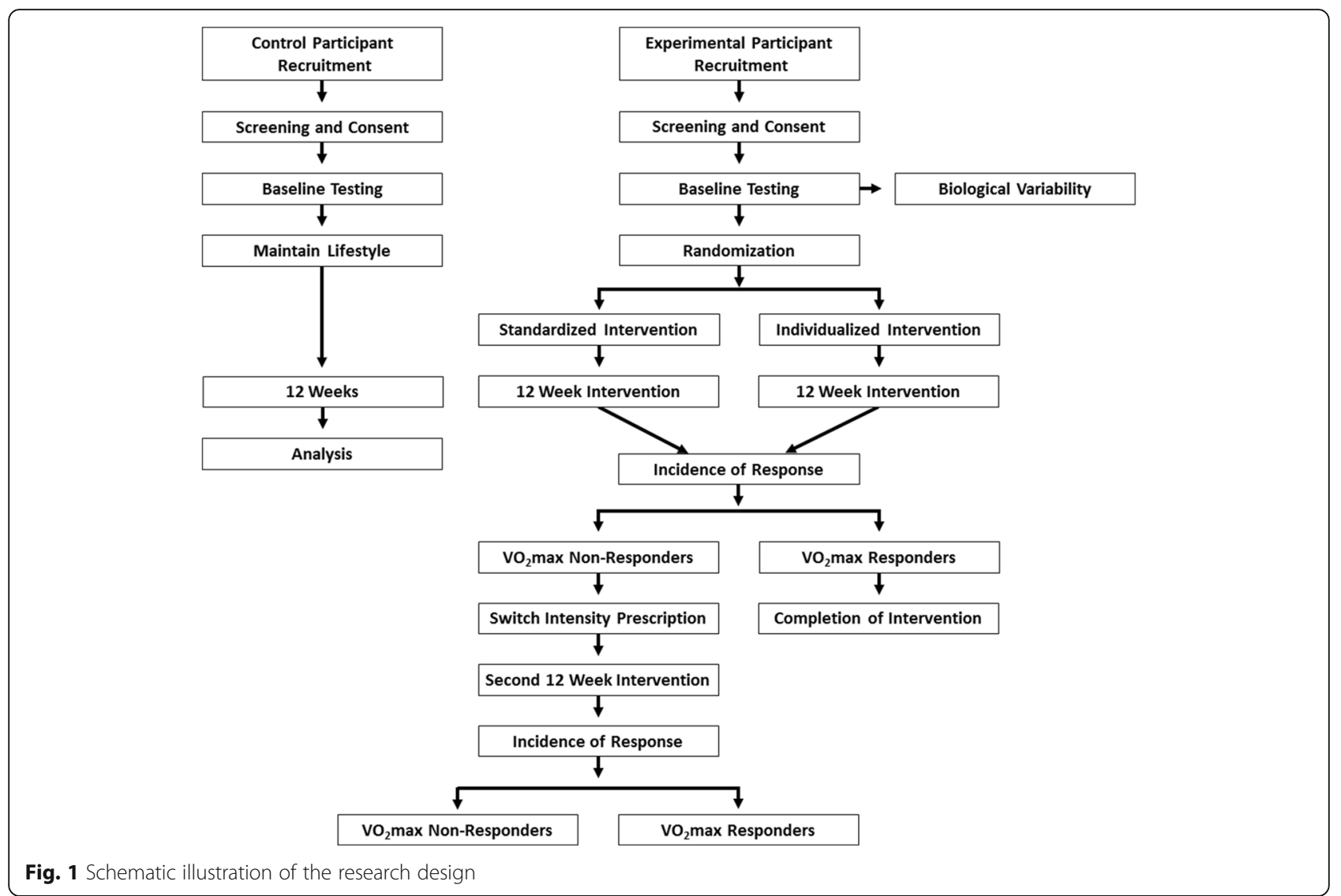

schedule or Tables 1, 2 and 3 for SPIRIT schedule design). If participants do not have a favorable outcome after 12 weeks (i.e. non-responders), they will complete a second 12-week intervention with a crossover design of the other exercise prescription. The protocol has been approved by the Auckland University of Technology Ethics Committee (16/264) and the Human Research Committee of the Institutional Review Board at Western State Colorado University (HRC2016-0190R6) with data collection occurring only at Western State Colorado University.

\section{Sample - experimental groups}

For the experimental groups, participants will be recruited from a community-based wellness program serving the local area. The wellness program participants are either referred by local medical professionals or seek entrance into the program from peer referrals. In order for participants to be included in the study, they must meet the following inclusion criteria:

- 30 to 75 years of age

- Considered low to moderate risk for cardiovascular disease [4]
- Currently sedentary (participating in less than 30 minutes of moderate intensity physical activity on at least 3 days a week)

- Resided at an altitude near $2300 \mathrm{~m}$ for at least the last 6 months

Participants will be excluded from the study if they have any signs, symptoms, or diagnosed cardiovascular, pulmonary, or metabolic disease. During the trial, participants will be asked to maintain their normal lifestyle to ensure any adaptations were due to the intervention.

\section{Sample - control group}

Due to moral and ethical issues related to excluding participants from an exercise intervention to improve health, a control group will be recruited as a separate cohort. The control group participants must meet the same inclusion criteria and will not be allowed in the study if they meet the exclusion criteria, as previously mentioned.

\section{Sample size calculation}

Sample size was projected with change in $\mathrm{VO}_{2} \max$ as the main outcome variable. The means and standard deviations of a previous study [5] were examined and 
Table 1 SPIRIT study calendar for the experimental group for the first 12-week intervention

\begin{tabular}{|c|c|c|c|c|c|c|c|}
\hline \multirow[b]{2}{*}{ Point of time: } & \multirow[b]{2}{*}{ Pre-intervention } & \multirow[b]{2}{*}{ Baseline } & \multicolumn{5}{|c|}{ Exercise intervention } \\
\hline & & & Time 0 & Week 4 & Week 8 & Week 12 & 12-week analysis \\
\hline \multicolumn{8}{|l|}{ Enrollment } \\
\hline Recruitment & $x$ & & & & & & \\
\hline Medical history & $x$ & & & & & & \\
\hline Inclusion criteria & $x$ & & & & & & \\
\hline Randomization & & & $x$ & & & & \\
\hline \multicolumn{8}{|l|}{ Testing } \\
\hline Height & & $x$ & & $x$ & $x$ & $x$ & \\
\hline Weight & & $x$ & & $x$ & $x$ & $x$ & \\
\hline Resting heart rate & & $x$ & & $x$ & $x$ & $x$ & \\
\hline Resting blood pressure & & $x$ & & $x$ & $x$ & $x$ & \\
\hline Waist circumference & & $x$ & & $x$ & $x$ & $x$ & \\
\hline Low-density lipoprotein & & $x$ & & $x$ & $x$ & $x$ & \\
\hline High-density lipoprotein & & $x$ & & $x$ & $x$ & $x$ & \\
\hline Triglycerides & & $x$ & & $x$ & $x$ & $x$ & \\
\hline Blood glucose & & $x$ & & $x$ & $x$ & $x$ & \\
\hline Maximal exercise test & & $x$ & & $x$ & $x$ & $x$ & \\
\hline Verification test & & $x$ & & $x$ & $x$ & $x$ & \\
\hline 3-day nutrition recall & & $x$ & & & & $x$ & \\
\hline \multicolumn{8}{|l|}{ Analysis } \\
\hline Biological variability & & & $x$ & & & & \\
\hline Time course changes & & & & & & & $x$ \\
\hline Incidence of response & & & & & & & $x$ \\
\hline
\end{tabular}

SPIRIT Standard Protocol Items: Recommendations for Interventional Trials

the effect size for this research study was calculated. Assuming a power of 0.80 was needed and the calculated effect size for change in $\mathrm{VO}_{2}$ max was 0.30 , it was determined that approximately 16 participants would be needed for each group [12]. It is assumed there would be an approximate $20 \%$ dropout rate, so the aim will be to achieve 20 participants per group.

\section{Intervention}

\section{Testing}

Testing sessions for both the experimental and control groups will be conducted at baseline and every fourth week during the 12-week intervention period. Testing sessions every fourth week will help to establish the current physiological levels to develop the exercise prescription for the experimental groups.

Testing will be conducted in a university-based performance laboratory under the supervision of three exercise physiologists. Prior to completing the testing sessions, participants will be asked to refrain from food and drink (other than water) for 12 hours prior to the testing session, be well hydrated prior, avoid the use of alcohol, caffeine, and tobacco within 24 hours of testing, be well rested, avoid significant exertion or exercise the day of testing, and report any medication use prior to testing. Testing will occur as close to the same time of day as possible with the above directions prior to each testing session. Following the blood lipid profile testing (explained in detail later) and prior to the maximal exercise test, participants will be provided a small snack. The testing will be conducted as follows:

Dietary analysis: participants will be instructed to not change their usual diets throughout the study and asked to complete a 3-day dietary intake recall including two weekdays and one weekend day to evaluate energy intake and the proportion of kilocalories from carbohydrates, protein, and fat. Anthropometric measurements: participants will be weighed on a calibrated, medical-grade scale to the nearest $0.01 \mathrm{~kg}$ and height will be measured using a stadiometer to the nearest $0.5 \mathrm{~cm}$. Waist circumference will be measured by the narrowest horizontal circumference above the umbilicus and below the xiphoid process to the nearest $0.5 \mathrm{~cm} \mathrm{[4]}$. 
Table 2 SPIRIT study calendar for the control group for the first 12-week intervention

\begin{tabular}{|c|c|c|c|c|c|c|c|}
\hline Point of time: & Pre-intervention & Baseline & Time 0 & Week 4 & Week 8 & Week 12 & 12-week analysis \\
\hline \multicolumn{8}{|l|}{ Enrollment } \\
\hline Recruitment & $x$ & & & & & & \\
\hline Medical history & $x$ & & & & & & \\
\hline Inclusion criteria & $x$ & & & & & & \\
\hline \multicolumn{8}{|l|}{ Testing } \\
\hline Height & & $x$ & & $x$ & $x$ & $x$ & \\
\hline Weight & & $x$ & & $x$ & $x$ & $x$ & \\
\hline Resting heart rate & & $x$ & & $x$ & $x$ & $x$ & \\
\hline Resting blood pressure & & $x$ & & $x$ & $x$ & $x$ & \\
\hline Waist circumference & & $x$ & & $x$ & $x$ & $x$ & \\
\hline Low-density lipoprotein & & $x$ & & $x$ & $x$ & $x$ & \\
\hline High-density lipoprotein & & $x$ & & $x$ & $x$ & $x$ & \\
\hline Triglycerides & & $x$ & & $x$ & $x$ & $x$ & \\
\hline Blood glucose & & $x$ & & $x$ & $x$ & $x$ & \\
\hline Maximal exercise test & & $x$ & & $x$ & $x$ & $x$ & \\
\hline Verification test & & $x$ & & $x$ & $x$ & $x$ & \\
\hline 3-day dietary recall & & $x$ & & & & $x$ & \\
\hline IPAQ & & $x$ & & $x$ & $x$ & $x$ & \\
\hline \multicolumn{8}{|l|}{ Analysis } \\
\hline 12-week changes & & & & & & & $x$ \\
\hline
\end{tabular}

SPIRIT Standard Protocol Items: Recommendations for Interventional Trials, IPAQ International Physical Activity Questionnaire

Table 3 SPIRIT study calendar (second 12 weeks) for $\mathrm{VO}_{2}$ max non-responders after the first 12-week intervention

\begin{tabular}{|c|c|c|c|c|c|c|c|}
\hline \multirow[b]{2}{*}{ Point of time: } & \multirow[b]{2}{*}{ Pre-intervention } & \multirow[b]{2}{*}{ Baseline } & \multicolumn{5}{|c|}{ Exercise intervention } \\
\hline & & & Time 0 & Week 4 & Week 8 & Week 12 & 12-week analysis \\
\hline \multicolumn{8}{|l|}{ Pre-intervention } \\
\hline Switch of experimental group & $x$ & & & & & & \\
\hline \multicolumn{8}{|l|}{ Testing } \\
\hline Height & & $x$ & & $x$ & $x$ & $x$ & \\
\hline Weight & & $x$ & & $x$ & $x$ & $x$ & \\
\hline Resting heart rate & & $x$ & & $x$ & $x$ & $x$ & \\
\hline Resting blood pressure & & $x$ & & $x$ & $x$ & $x$ & \\
\hline Waist circumference & & $x$ & & $x$ & $x$ & $x$ & \\
\hline Low-density lipoprotein & & $x$ & & & & & \\
\hline High-density lipoprotein & & $x$ & & & & & \\
\hline Triglycerides & & $x$ & & & & & \\
\hline Blood glucose & & $x$ & & & & & \\
\hline Maximal exercise test & & $x$ & & $x$ & $x$ & $x$ & \\
\hline Verification test & & $x$ & & $x$ & $x$ & $x$ & \\
\hline 3-day nutrition recall & & $x$ & & & & $x$ & \\
\hline \multicolumn{8}{|l|}{ Analysis } \\
\hline Incidence of response & & & & & & & $x$ \\
\hline
\end{tabular}


Resting heart rate (RHR) and BP measurements: procedures for RHR and BP will follow standard guidelines [4]. In summary, participants will be required to sit for 5 minutes with sufficient back support, feet on the ground, and arms supported at heart level. Resting heart rate will be recorded by using a medicalgrade pulse oximeter after the 5 minutes of seated rest. Blood pressure will be measured using a stethoscope and sphygmomanometer to determine left arm brachial artery BP on consecutive measure separated by 1 minute. The mean of the systolic and diastolic measures will be considered the resting BP.

Fasting blood glucose and lipid measurements: all fasting lipid and blood glucose measurements will be analyzed using the Cholestech LDX system (Alere, Waltham, MA, USA), which has been shown to have excellent reproducibility $[13,14]$. An optics check of the Cholestech LDX system will be completed at the beginning of each testing session. Participants will be asked to thoroughly wash hands with soap and rinse with warm water. The skin will then be wiped with an alcohol swab and allowed to dry. Using a lancet, the distal end of the third digit of the right hand will be punctured and a finger stick sample will be collected using a $40 \mu \mathrm{l}$ capillary tube with blood flowing freely into the tube without milking the finger. The blood sample will then be extracted into a commercially available test cassette for analysis. Measurements of TC, HDL, LDL, triglycerides, and blood glucose will be obtained. Upon completion of the blood profile testing and data collection, blood samples will be disposed of based on standard biohazard procedures.

$\mathrm{VO}_{2}$ max and verification bout: participants will complete a modified Balke, pseudo-ramp graded exercise test (GXT) on a power treadmill. Participants will walk or jog at a self-selected pace with an increase in incline of $1 \%$ every minute until volitional fatigue. Heart rate and expired gas will be measured continuously using a heart rate monitor and a calibrated metabolic analyzer, respectively. Data will be analyzed following guidelines previously reported [5]. In summary, gas exchange data will be time averaged for every 15 seconds, $\mathrm{VO}_{2}$ max will be determined by averaging the last two 15 second samples, and maximal HR will be the highest achieved HR during the GXT.

Since a verification procedure has been found to be effective in middle-aged and older adults to confirm $\mathrm{VO}_{2} \max [15]$, this procedure will be used to ensure participants have reached maximal capacity. The verification trial will be performed 20 minutes after the GXT as recommended elsewhere [16] and has been confirmed to be an effective procedure at altitude [17]. The verification bout will consist of a workload that is $105 \%$ of the maximal workload during the GXT (last fully completed stage) as this workload has been shown to be sufficient to elicit verification test durations of $2-3$ minutes $[15,18]$ and will continue until volitional fatigue. Analysis of the verification bout will follow the same protocol as the GXT. 'True' $\mathrm{VO}_{2} \max$ will be considered to be attained if the GXT and verification bout are within $\pm 3 \%$ [15]. If participants are unable to reach $\mathrm{VO}_{2} \max$, they will be asked to repeat the trial no sooner than $24 \mathrm{~h}$ later.

The control group will be asked to maintain their normal lifestyle activity habits. Therefore, in addition to the previously stated battery of testing, control participants will also complete the International Physical Activity Questionnaire (IPAQ) every fourth week at the testing session.

\section{Biological variability and technical error}

In order to establish criteria to categorize participants as responders or non-responders, the biological variability will be established to determine the TE. Therefore, from the pool of experimental research participants, 15 participants will be randomly selected based on when the referral or inquiry into the wellness program is received (i.e., participants $1,3,5,7$, etc. until 15 confirmed participants have been reached. If there are not 15 participants after the first round, then participants $2,4,6$, etc. will be asked until the desired total of 15 participants is met). Participants will be asked to complete the baseline testing assessments twice within a 2-week period to determine the day-to-day biological variability. The biological variability will be combined with the typical error of the equipment utilized (sourced from the literature and company of the equipment) to determine the TE. More details related to the statistical approaches are located in the statistical analysis section.

\section{Exercise intervention}

After the completion of the baseline testing, participants will be randomly allocated to either the individualized or standardized arms at a 1:1 ratio using a computerized stratified minimization sequence. One of the primary investigators will have knowledge of the treatment groups to which participants have been allocated in order to interpret test results and prescribe target exercise intensities. However, this same investigator will not be involved in the implementation of the exercise training programs (to be completed by research assistants) in order to mitigate researcher bias. Participants will then be asked to come to the laboratory on Monday, Wednesday, and Friday to take part in the community wellness program and subsequent research. Upon arrival each day, participants will be asked to rest comfortably for 5 minutes in the seated position. Then, their resting 
BP and HR will be recorded. Following the resting measurements, participants will complete a 5-minute warm-up starting at a low and progressively increasing intensity until they are ready to begin their CRF exercise session. At this point, participants will be asked to stay within the designated HR (described in further detail below) outlined on their exercise log as determined based on their experimental group and week of experimental trial. At approximately $1 / 3$ and $2 / 3$ the total session time, an exercise physiologist or research assistant will record their current $\mathrm{HR}$, rating of perceived exertion (scale 1-10), intensity of aerobic equipment, and any other pertinent notes. At the end of the CRF exercise session, the participant will be asked to complete a cooldown in which the exercise intensity is gradually reduced. While resistance training is not part of this proposed experiment, it is an integral part of the community wellness program and could be a confounding factor to the overall incidence of response. Therefore, all participants will be asked to complete the resistance training after the CRF training session is completed in order to have consistency among all participants. During the first 4 weeks, there will be no resistance training. During the next 4 weeks (week 4-8), there will be a learning and anatomical adaptation phase to resistance training in which proper technique and range of motion will be emphasized and participants will be acclimated to the resistance training machines. During the last 4 weeks (week 8-12) participants will complete one set of 8-12 repetitions on eight machine-based resistance training exercises and progress to two sets by the end of the 12th week [19].

\section{Determination of workload}

For the standardized group, the workload will be determined based on \%HRR and completed based on the following calculation:

HRR $=[($ Maximal HR-Resting HR $) \times$ Desired Percentage $]+$ Resting HR

For the individualized group, the workload will be determined based on ventilatory threshold values as previously described $[5,20]$ to determine VT1 and VT2. The criteria used for determining VT1 and VT2 will be a visual analysis of figures of time plotted against the relative respiratory variable - ventilatory equivalents of oxygen $\left(\mathrm{VE} / \mathrm{VO}_{2}\right)$ and ventilatory equivalents of carbon dioxide $\left(\mathrm{VE} / \mathrm{VCO}_{2}\right)$. Determination of $\mathrm{VT} 1$ will be an increase in $\mathrm{VE} / \mathrm{VO}_{2}$ with no increase in $\mathrm{VE} / \mathrm{VCO}_{2}$ and moving away from linearity of VE, whereas VT2 will be a simultaneous increase in both $\mathrm{VE} / \mathrm{VO}_{2}$ and $\mathrm{VE} / \mathrm{VCO}_{2}$. Calculations of $\mathrm{HR}$ values associated with ventilatory threshold (VT) values will be calculated prior to exercise sessions and with following HR ranges:
- Target $\mathrm{HR}>\mathrm{VT} 1=\mathrm{HR}$ range of $10 \mathrm{bpm}$ below VT1 to the HR at VT1

- Target $\mathrm{HR} \geq \mathrm{VT} 1$ to $<\mathrm{VT} 2=\mathrm{HR}$ range of $15 \mathrm{bpm}$ above VT1 and below VT2

- Target $\mathrm{HR} \geq \mathrm{VT} 2=\mathrm{HR}$ range of $10 \mathrm{bpm}$ above VT2

Exercise volume will be prescribed based on energy expenditure per $\mathrm{kg}$ of body weight a week $\left(\mathrm{kcal} \cdot \mathrm{kg}^{-1}\right.$. week ${ }^{-1}$ ) to implement an isocaloric exercise volume (i.e., in terms of kilocalories [kcal] per $\mathrm{kg}$ a week) across individuals and groups. Previous research has found that energy expenditure ranging from $4 \mathrm{kcal} \cdot \mathrm{kg}^{-1} \cdot$ week $^{-1}$ [21] to $23 \mathrm{kcal} \cdot \mathrm{kg}^{-1} \cdot$ week $^{-1}$ [22-25] have positive effects on CRF and cardiometabolic responses to exercise. Therefore, this study will utilize a similar 12-week exercise protocol as previously described [5], while implementing a standardized isocaloric volume $\left(\mathrm{kcal} \cdot \mathrm{kg}^{-1} \cdot\right.$ week $\left.^{-1}\right)$ instead of a designated time for each exercise session. Exercise progression will follow standard guidelines that have been previously established [4]. Figure 2 illustrates the exercise progression following baseline testing through the 12-week intervention for both experimental groups while Tables 1 and 2 show the SPIRIT study schedule for experimental and control participants, respectively.

\section{Time course changes over 12 weeks}

Currently, there is no literature investigating the time course changes of $\mathrm{VO}_{2} \mathrm{max}$ and cardiometabolic risk factor values between a standardized and individualized CRF exercise program. Therefore, since testing will occur every fourth week, the time course changes over the first 12 weeks of the CRF training intervention will be highlighted and addressed. These data will help further analyze the incidence of response during 12 weeks of structured CRF training and gain insight into the time course changes associated with CRF training for each individual and based on the CRF training intensity (standardized or individualized).

\section{Categorizing responders and non-responders}

Following the 12-week intervention, the participants will be categorized as responders and non-responders (see statistical analysis section for further details) for each testing measurement. While each testing measurement will be evaluated, the overreaching categorization of responder and non-responder will be based on $\mathrm{VO}_{2} \max$ due to the profound health and performance implications of this measurement.

\section{Modifying the prescription of non-responders}

For the participants that are categorized as responders after the initial 12 weeks, they will be completed with the study. However, participants that were non-responders 


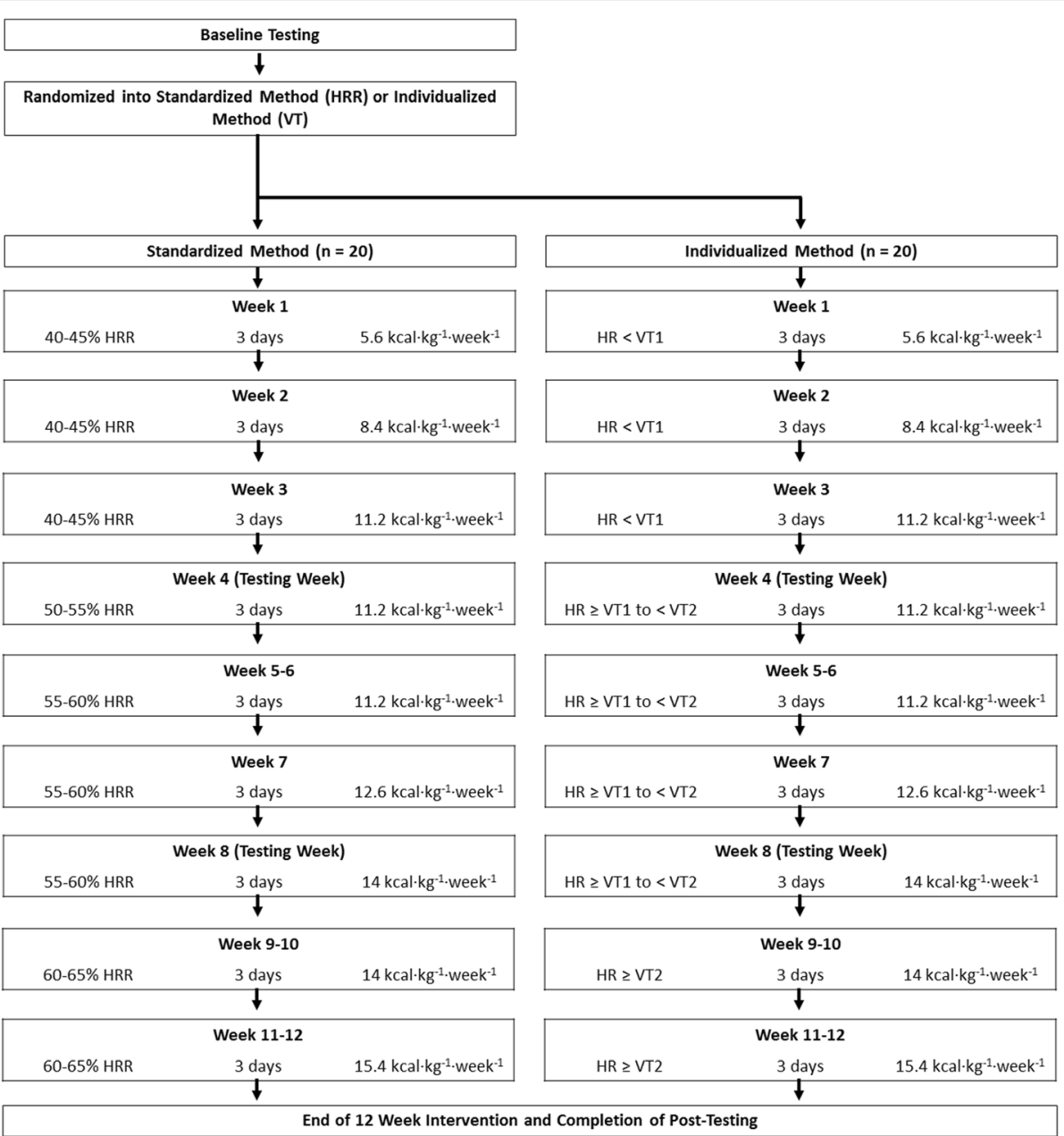

Fig. 2 A detailed flow schematic of the exercise prescription for the experimental groups. HRR heart rate reserve, $\mathrm{kcal}$ kilocalories, $\mathrm{kg}$ kilograms, VT1 first ventilatory threshold, VT2 second ventilatory threshold

based on $\mathrm{VO}_{2}$ max changes will be asked to complete a subsequent 12-week intervention. During this second 12week intervention, participants will switch their exercise intensity prescription (i.e., if a participant in the standardized group was categorized as a non-responder, they will complete the second 12 weeks in the individualized group). The experimental design including the exercise prescription and testing (baseline and post-testing) will follow the same schedule and protocol. However, during week 4 and 8 the testing sessions will not include blood analysis. The SPIRIT study schedule for the second 12week intervention can be seen in Table 3.

At the completion of the second 12-week intervention, the participants will again be categorized as responders or non-responders based on the new baseline and 12week testing values. This crossover design intervention will help to gain insight on whether or not 'non-responders' can become responders if the intensity of the exercise prescription is modified.

\section{Data and confidentiality management}

Electronic data will be coded, entered, and stored into a secure (password-protected) database on Western State Colorado University's campus. All paper data, including consent forms, medical history documents, and daily exercise logs, will be stored in a secured locked cabinet in the Western State Colorado University Human Subject's office. Only the primary investigators will have access to the data.

Due to the nature of the study, participants will be exercising in an environment with other members from the study. Therefore, anonymity of identity cannot be guarantee throughout the study. However, no research participant will be able to see or access any personal information - medical documents, exercise log, medications, etc. To ensure participant safety when exercising, researcher assistants delivering the exercise will be informed of relevant information that may influence how the participant responds to exercise. Any data collected 
and displayed in results of scientific manuscripts will be displayed in a way which does not disclose individual identity.

\section{Statistical analysis}

All analyses will be performed using IBM SPSS Version 22.0 (Armonk, NY, USA) and GraphPad Prism 6.0 (GraphPad Software, San Diego, CA, USA).

\section{Biological variability}

Intraclass correlation (ICC) of variation, typical error and co-efficient of variation $(\mathrm{CV})$ for $\mathrm{VO}_{2} \mathrm{max}$, resting $\mathrm{HR}$, resting $\mathrm{BP}$, and fasting blood glucose, total cholesterol, HDL, and LDL will be calculated as described previously [26]. The CV will be combined with the measurement error of the testing to determine the TE. The TE will be used to categorize responders and nonresponders. In summary, for all criteria tested the changes in pre- to post- intervention will be analyzed with responders having a change $>\mathrm{TE}$ and non-responders having a change that is $\leq \mathrm{TE}$.

\section{Time course changes}

Measures of centrality and spread will be presented as mean \pm standard deviation (SD) and ranges will be reported for each measure. The precision of estimates will be reported as $90 \%$ confidence limits. Baseline-dependent variables will be compared using general linear model (GLM) ANOVA and Tukey post hoc tests, when applicable. Because baseline and every fourth week program data will be available after the completion of the first 12 weeks the effect of training on CRF $\left(\mathrm{VO}_{2} \max \right)$ and cardiometabolic measurements (TC, HDL, LDL, resting $\mathrm{BP}$, and resting $\mathrm{HR}$ ) will be determined using repeatedmeasures GLM-ANOVA with the exercise intensity (individualized or standardized) as the between subjects factor. Between group 12-week changes will be analyzed using GLM-ANOVA and Tukey post hoc tests, when appropriate. The assumption of normality will be tested by examining normal plots of the residuals in ANOVA models and will be regarded as normally distributed if Shapiro-Wilk tests are not significant [12]. Effect sizes will be calculated using means and pooled standard deviations. Method of data analysis will be analysis by treatment administered. Only participants who complete $\geq 70 \%$ exercise training sessions and strictly adhere to target exercise intensity will be included in the statistical analysis. The probability of making a type I error will be set at $p \leq 0.05$ for all statistical analyses.

\section{Incidence of response}

Delta values $(\Delta)$ will be calculated (post-testing minus baseline value divided by baseline value) for percent change in relative $\mathrm{VO}_{2}$ max, TC, HDL, LDL, resting BP and resting $\mathrm{HR}$ and participants will be categorized as: ' 1 ' = responder $(\% \Delta>\mathrm{TE})$ or ' 0 ' $=$ non-responder $(\% \Delta \leq$ TE). Chi-squared $\left(\chi^{2}\right)$ tests will be subsequently used to analyze the point prevalence of responders and nonresponders to exercise training separated by exercise intensity group (individualized and standardized) between baseline and the end of the end the first 12-week intervention. Only participants who complete $\geq 70 \%$ exercise training sessions and strictly adhere to target exercise intensity will be included in the statistical analysis. The probability of making a type I error will be set at $p \leq$ 0.05 for all statistical analyses.

In order to make inferences about the true values (population values) of the effect of both exercise interventions on $\mathrm{VO}_{2} \mathrm{max}$ and cardiometabolic factors, the uncertainty in effect will be expressed as $90 \%$ confidence limits and the likelihood the true value of the effect represents a substantial and clinically meaningful change (harm or benefit). Effects will be declared unclear if the confidence interval overlapped thresholds for substantiveness or the effect could be substantially positive and negative or beneficial and detrimental. All probabilistic magnitude-based inferences will be calculated using a published spreadsheet [27].

\section{Discussion}

There has been a considerable amount of individual variability reported in the literature related to the response of CRF measurements (specifically, $\mathrm{VO}_{2} \max$ and peak aerobic ability $\left[\mathrm{VO}_{2}\right.$ peak]). However, there is still an overall lack of understanding as to why this variability occurs. Unfortunately, there is minimal consistency in methodology and the criteria for determining incidence of response leading to the overall findings indicating there are changes in training responsiveness of -33 to $+76 \%$ [7]. However, some of the data associated with individual responses may be misleading as measurements were recorded as peak values $[8,28,29]$ and may not be a direct representation of the maximal efforts for participants and, therefore, not an accurate representation of true physiological adaptations.

In order to have an all-inclusive definition for incidence of response the TE must be taken into account [10]. Therefore, it would be important to know biological variability and measurement error for each outcome to determine whether responses are beyond that of the TE. Two recent investigations $[5,30]$ utilized TE to determine response rate by defining a responder as an individual with improvements from pre- to posttraining by $>\mathrm{TE}$ in a positive direction and, in contrast, an individual who improves by $\leq \mathrm{TE}$ as a non-responder. Nevertheless, for the two aforementioned studies, values for day-to-day biological variability were used from previously published work and may not be directly applicable 
to the population being studied or the environmental conditions in which data collection takes place.

Conventionally, results of exercise-based studies are reported as the mean and standard deviation [31] and only illustrate the main effects and group differences of training responsiveness [32]. Overall, there is a lack in attention to individual differences with these conventional methods of reporting data since nearly $32 \%$ of measurements (distributed normally) fall outside of one standard deviation. Recent literature proposes reporting not only the mean, standard deviation, and group differences, but also individual responses to the training program [31, 32] or at least ranges of endurance changes [30]. This approach will strengthen study findings and provide further insight into the phenomenon of individual variability and training responsiveness.

From the HERITAGE Family Study [33], a large, wellcontrolled, 20-week standardized endurance training program, insight was gained on the incidence of response. It was reported that genetics may play a critical role in the incidence of response [34] with trainability of $\mathrm{VO}_{2}$ max linked to familial aggregation [35]. However, a potentially overlooked factor in the individual variability may be linked to poor methodology of exercise prescription. Indeed, due to the theoretical and physiological mechanisms of exercise prescription, utilization of a threshold-based measurement for exercise prescription has been suggested to decrease the incidence of nonresponse and improve CRF and cardiometabolic factors compared to the traditional approach using intensities set relative to $\mathrm{VO}_{2} \max , \mathrm{HRmax}, \mathrm{VO}_{2} \mathrm{R}$, or HRR [36]. However, there have been few studies that have reported individual responses following training relative to a threshold measurement [31]. To the best of our knowledge, there is currently only one study reporting individual responses to training comparing set intensities based on the first ventilatory threshold (VT1) and the second ventilatory threshold (VT2) measurements and percentage of HRR [5]. During incremental exercise, VT1 is the point at which increases in ventilation become non-linear (an increase in the ventilatory equivalents of oxygen $\left[\mathrm{VE} / \mathrm{VO}_{2}\right]$ with no increase in the ventilatory equivalents of carbon dioxide $\left[\mathrm{VE} / \mathrm{VCO}_{2}\right]$ ) and VT2 is the point at which there is an accumulation of blood lactate due to the inability to buffer the amount of lactate produced (simultaneous increase in both $\mathrm{VE} / \mathrm{VO}_{2}$ and $\mathrm{VE} / \mathrm{VCO}_{2}$ ) [37].

Traditionally, exercise intensity has been prescribed based on a relative percent concept - based on a percentage of $\mathrm{HRmax}, \mathrm{VO}_{2} \max , \mathrm{VO}_{2} \mathrm{R}$, or HRR. However, caution has been advised for utilization of the relative percent method, specifically HRmax and $\mathrm{VO}_{2} \max$, as criteria to determine workload as they may not be sufficient to elicit the desired metabolic response [3, 38]. Furthermore, percentages for both HRmax and $\mathrm{VO}_{2}$ max correspond to a wide range of exercise intensities relative to threshold measurements [39]. For example, with exercise intensities between $58 \%$ and $75 \%$ of $\mathrm{VO}_{2} \max$, some participants were found to be above while others were reported to be below their individual anaerobic threshold [40]. Similar findings were noted when investigating a 12-month jogging/walking program [38]. In order to make the prescription of exercise intensity more individualized, many researchers have used percentages of HRR as this takes into consideration not only HRmax, but also resting HR. However, aerobic thresholds were found to be at $70 \% \pm 10 \%$ of HRR [41] indicating large variability in the metabolic stress across individuals at a set percentage of HRR.

Indeed, genetics have gained a lot of attention to understand the specific roles of genes and response rates. However, based on the genetic evidence to date, there are many pathways that are associated with $\mathrm{VO}_{2}$ max trainability and nearly an unlimited combination of signaling events that may influence the $\mathrm{VO}_{2}$ max responsiveness [42, 43]. With genetics proposed to account for less than $50 \%$ of the variance in responsiveness, the other $50 \%$ is still not well understood.

One of the major areas in which the literature is lacking in the understanding of training responsiveness is the investigation of an individualized approach to exercise prescription and the time course changes. With the emerging concept of 'exercise is medicine' and the capacity to prescribe exercise to combat adverse effects of disease, the time course changes for $\mathrm{VO}_{2}$ max and cardiometabolic risk factor measures need to be better understood to properly identify efficacious exercise doses (i.e., intensity, volume) that will elicit an adequate response. However, much of the literature on time course changes utilizes standardized methods of exercise prescription rather than individualized approaches. To the best of our knowledge, there is no literature in time course changes of $\mathrm{VO}_{2} \mathrm{max}$ and cardiometabolic risk factor outcomes with the use of a thresholdbased protocol and exercise volume individualized based on kilocalories of expenditure per week with relation to body mass. Similarly, results of time course changes have traditionally been reported as only group means and standard deviations with individual time course changes not being reported. Based on a review of the literature, there have only been two studies to identify individual time course changes $[44,45]$. Reporting of individual time course changes for $\mathrm{VO}_{2} \max$ and cardiometabolic risk factor measurements will help to further understand the individual variability in training responsiveness.

\section{Limitations}

There are several limitations that merit discussion. It is possible there may be heterogeneity in training responses due to age alone given the large age range (30 to 75 years) 
that will be recruited for the current trial. However, the age range for the target sample will be comparable to previous studies $[22,23,33]$ and also reflect the likely age range found in community exercise programs [46]. Another possible limitation is external validity given data collection will take place at moderate altitude. Nevertheless, to the best of our knowledge, there is no evidence to suggest differences in training responsiveness (i.e. responders and non-responders) between altitude-residing individuals and sea level counterparts. A third potential limitation is the inability to anticipate how many participants will be categorized as non-responders following the first 12-week intervention. Indeed, this limitation could arguably be the most significant due to the second part of the trial (crossing over non-responders to the other prescription group) being underpowered if there are a low number of non-responders. Nevertheless, to our knowledge, there are no previous investigations evaluating a crossover design to determine if non-responders can become responders with a different exercise prescription protocol. Therefore, even if the second part of the trial is underpowered, the results would provide valuable preliminary insight into the efficacy of individualized exercise prescription.

In summary, this original randomized controlled trial aims to (1) investigate the efficacy of an individualized exercise prescription at improving training responsiveness and, (2) to better understand the time course changes of training adaptations to both individualized and standardized exercise intensity prescription methods. It is anticipated that findings from this novel trial will add to our knowledge of how personalized exercise can enhance training efficacy and limit training unresponsiveness.

\section{Study status}

Recruitment will commence in September of 2016. The estimated completion date is mid-late 2018.

\section{Additional files}

Additional file 1: SPIRIT checklist. (DOC $120 \mathrm{~kb}$ )

Additional file 2: Overall SPIRIT study schedule. (PDF 231 kb)

\footnotetext{
Abbreviations

BP: blood pressure; CRF: cardiorespiratory fitness; CV: co-efficient of variation; GLM: general linear model; GXT: graded exercise test; HDL: high-density lipoprotein; HR: heart rate; HRR: heart rate reserve; ICC: intra-class correlation; IPAQ: International Physical Activity Questionnaire; kcal: kilocalorie; LDL: lowdensity lipoprotein; RHR: resting heart rate; SD: standard deviation; SPIRIT: Standard Protocol Items: Recommendations for Interventional Trials; TC: total cholesterol; TE: technical error; $V E N \mathrm{NO}_{2}$ : ventilatory equivalents of carbon dioxide; $\mathrm{VE} \mathrm{NO}_{2}$ : ventilatory equivalents of oxygen; $\mathrm{VO}_{2}$ max: maximal oxygen uptake; $\mathrm{VO}_{2}$ peak: peak aerobic ability; $\mathrm{VO}_{2} \mathrm{R}$ : aerobic ability reserve; $\mathrm{VT}$ : ventilatory threshold; VT1: first ventilatory threshold; VT2: second ventilatory threshold
}

\section{Acknowledgements}

Not applicable.

\section{Funding}

This study will be supported by Professional Activity Fund grants to RW and LD from Western State Colorado University. The funding body (Western State Colorado University) was not involved in development of the study design or preparation of the manuscript. Nor will they be involved with subsequent data collection and analysis.

Availability of data and materials

Not applicable.

\section{Authors' contributions}

$\mathrm{RW}, \mathrm{LD}, \mathrm{NH}$, and AK contributed to conception and design of the experiment. RW, LD, NH, and AK contributed to preparation of the manuscript. All authors read and approved the final manuscript.

\section{Authors' information}

Not applicable.

\section{Competing interests}

The authors declare that they have no competing interests.

\section{Consent for publication}

Not applicable.

\section{Ethics approval and consent to participate}

The protocol has been approved by the Auckland University of Technology Ethics Committee (16/264) and the Human Research Committee of the Institutional Review Board at Western State Colorado University (HRC201601-90R6). It should be noted that even though two academic institutions reviewed and approved the protocol, data collection will only be occurring on the campus of Western State Colorado University. Prior to any data collection and after all questions participants have about the study protocol, time commitment, and expectations have been answered, an informed consent document will be signed. If the informed consent is not signed, the participant will be excluded from the data collection.

\section{Author details}

${ }^{1}$ Auckland University of Technology, Human Potential Centre, Auckland, New Zealand. ${ }^{2}$ Auckland University of Technology, Sports Performance Research Institute New Zealand, Auckland, New Zealand. ${ }^{3}$ Department of Recreation, Exercise, and Sport Science, Western State Colorado University, Gunnison, $\mathrm{CO}, \mathrm{USA}$.

Received: 24 August 2016 Accepted: 28 November 2016

Published online: 19 December 2016

\section{References}

1. Bouchard C. Human adaptability may have a genetic basis. In: Landry F, editor. Health, risk estimation, risk reduction and health promotion. Proceedings of the 18th Annual Meeting of the Society of Prospective Medicine. Ottawa: Canadian Public Health Association; 1983. p. 463-76.

2. Lortie G, Simoneau J, Hamel P, Boulay M, Landry F, Bouchard C. Responses of maximal aerobic power and capacity to aerobic training. Int J Sports Med. 1984;5:232-6.

3. Katch V, Weltman A, Sady S, Freedson P. Validity of the relative percent concept for equating training intensity. Eur J Appl Physiol. 1978;39:219-27.

4. American College of Sports Medicine. ACSM's quidelines for exercise testing and prescription. 9th ed. Baltimore: Lippincott Williams \& Wilkins; 2014.

5. Wolpern AE, Burgos DJ, Janot JM, Dalleck LC. Is a threshold-based model a superior method to the relative percent concept for establishing individual exercise intensity? A randomized controlled trial. BMC Sports Sci Med Rehab. 2015:7:1-9.

6. Vollaard NBJ, Constantin-Teodosiu D, Fredriksson K, Rooyackers O, Jansson E, Greenhaff PL, et al. Systematic analysis of adaptations in aerobic capacity and submaximal energy metabolism provides a unique insight into determinants of human aerobic performance. J Appl Physiol. 2009;106:1479-86. 
7. Sisson SB, Katzmarzyk PT, Earnest CP, Bouchard C, Blair SN, Church TS. Volume of exercise and fitness non-response in sedentary, postmenopausal women. Med Sci Sports Exerc. 2009;41:539-45.

8. Chmelo EA, Crotts CI, Newman JC, Brinkley TE, Lyles MF, Leng X, et al. Heterogeneity of physical function responses to exercise training in older adults. J Am Geriatr Soc. 2015;63:462-9.

9. McPhee JS, Williams AG, Degens $H$, Jones DA. Inter-individual variability in adaptation of the leg muscles following a standardised endurance training programme in young women. Eur J Appl Physiol. 2010;109:1111-8.

10. Bouchard C, Blair SN, Church TS, Earnest CP, Hagberg JM, Häkkinen K, et al. Adverse metabolic response to regular exercise: is it a rare or common occurrence? PLoS One. 2012;7:e37887.

11. Chan AW, Tetzlaff JM, Gøtzsche PC, Altman DG, Mann H, Berlin JA, et al. SPIRIT 2013 explanation and elaboration: guidance for protocols of clinical trials. Brit Med J. 2013:346:e7586.

12. Cohen J. Statistical power analysis for the behavioral sciences. 2nd ed. Hillsdale: Lawrence Erlbaum Associates; 1988.

13. Shephard MD, Mazzachi BC, Shephard AK. Comparative performance of two point-of-care analysers for lipid testing. Clin Lab. 2007;53:561-6.

14. Dale RA, Jensen LH, Krantz MJ. Comparison of two point-of-care lipid analyzers for use in global cardiovascular risk assessments. Ann Pharmacother. 2008;42:633-9.

15. Dalleck LC, Astorino TA, Erickson RM, McCarthy CM, Beadell AA, Botten BH. Suitability of verification testing to confirm attainment of VO2max in middle-aged and older adults. Res Sports Med. 2012;20:118-28.

16. Nolan $P$, Beaven $M$, Dalleck $L$. Comparison of intensities and rest periods for VO2max verification testing procedures. Int J Sports Med. 2014:35:1024-9.

17. Weatherwax R, Richardson T, Beltz N, Nolan P, Dalleck L. Verification testing to confirm VO2max in altitude-residing, endurance-trained runners. Int J Sports Med. 2016:37:525-30.

18. Astorino T, White A, Dalleck L. Supramaximal testing to confirm attainment of VO2max in sedentary men and women. Int J Sports Med. 2009:30:279-84.

19. Garber CE, Blissmer B, Deschenes MR, Franklin BA, Lamonte MJ, Lee IM, et al. Quantity and quality of exercise for developing and maintaining cardiorespiratory, musculoskeletal, and neuromotor fitness in apparently healthy adults: guidance for prescribing exercise. Med Sci Sports Exerc. 2011:43:1334-59.

20. Wasserman K, Mcllroy MB. Detecting the threshold of anaerobic metabolism in cardiac patients during exercise. Am J Cardiol. 1964;14:844-52.

21. Church TS, Earnest CP, Skinner JS, Blair SN. Effects of different doses of physical activity on cardiorespiratory fitness among sedentary, overweight or obese postmenopausal women with elevated blood pressure: a randomized controlled trial. JAMA. 2007;297:2081-91.

22. Dalleck L, Van Guilder G, Richardson T, Vella C. The prevalence of adverse cardiometabolic responses to exercise training with evidence-based practice is low. Diab Metab Syndr Obes. 2015;8:73-8.

23. Kraus WE, Torgan CE, Duscha BD, Norris J, Brown SA, Cobb FR, et al. Studies of targeted risk reduction intervention through defined exercise (STRRIDE). Med Sci Sports Exerc. 2001;33:1774-84.

24. Lee MG, Park KS, Kim DU, Choi SM, Kim HJ. Effects of high-intensity exercise training on body composition, abdominal fat loss, and cardiorespiratory fitness in middle-aged Korean females. Appl Physiol Nutr Metab. 2012;37:1019-27.

25. Slentz CA, Duscha BD, Johnson JL, Ketchum K, Aiken LB, Samsa GP, et al. Effects of the amount of exercise on body weight, body composition, and measures of central obesity: Strride — a randomized controlled study. Arch Intern Med. 2004;164:31-9.

26. Hopkins WG. Measures of reliability in sports medicine and science. Sports Med. 2000;30:1-15.

27. Batterham AM, Hopkins WG. Making meaningful inferences about magnitudes. Int J Sports Physiol Perform. 2006;1:50-7.

28. Hautala AJ, Kiviniemi AM, Mäkikallio TH, Kinnunen H, Nissilä S, Huikuri HV, et al. Individual differences in the responses to endurance and resistance training. Eur J Appl Physiol. 2006;96:535-42

29. Leifer ES, Brawner CA, Fleg JL, Kraus WE, Whellan DJ, PiñA IL, et al. Are there negative responders to exercise training among heart failure patients? Med Sci Sports Exerc. 2014;46:219-24.

30. Scharhag-Rosenberger F, Walitzek S, Kindermann W, Meyer T. Differences in adaptations to 1 year of aerobic endurance training: individual patterns of nonresponse. Scand J Med Sci Sports. 2012;22:113-8.

31. Mann TN, Lamberts RP, Lambert MI. High responders and low responders: factors associated with individual variation in response to standardized training. Sports Med. 2014;44:1113-24.
32. Buford TW, Roberts MD, Church TS. Toward exercise as personalized medicine. Sports Med. 2013:43:157-65.

33. Bouchard C, Leon AS, Rao DC, Skinner JS, Wilmore JH, Gagnon J. The HERITAGE family study: Aims, design, and measurement protocol. Med Sci Sports Exerc. 1995;27:721-9.

34. Bouchard C. Genomic predictors of trainability. Exp Physiol. 2012;97:347-52.

35. Bouchard C, An P, Rice T, Skinner JS, Wilmore JH, Gagnon J, et al. Familial aggregation of $\mathrm{VO} 2 \mathrm{max}$ response to exercise training: results from the HERITAGE Family Study. J Appl Physiol. 1999:87:1003-8.

36. Mann T, Lamberts RP, Lambert MI. Methods of prescribing relative exercise intensity: physiological and practical considerations. Sports Med. 2013;43:613-25

37. Porcari J, Bryant C, Comana F. Exercise physiology. 1st ed. Philadelphia: F.A. Davis Company; 2015

38. Scharhag-Rosenberger F, Meyer T, Gäßler N, Faude O, Kindermann W. Exercise at given percentages of VO2max: heterogeneous metabolic responses between individuals. J Sci Med Sport. 2010;13:74-9.

39. Meyer T, Gabriel HHW, Kindermann W. Is determination of exercise intensities as percentages of VO2max or HRmax adequate? Med Sci Sports Exerc. 1999:31:1342-5.

40. Dwyer J, Bybee R. Heart rate indicies of the anaerobic threshold. Med Sci Sports Exerc. 1983:15:72-6.

41. Azevedo LF, Perlingeiro PS, Brum PC, Braga AMW, Negrão CE, de Matos LDNJ. Exercise intensity optimization for men with high cardiorespiratory fitness. J Sport Sci. 2011;29:555-61.

42. Hoppeler H. Molecular networks in skeletal muscle plasticity. J Exp Biol. 2016:219:205-13.

43. Sarzynski MA, Ghosh S, Bouchard C. Genomic and transcriptomic predictors of response levels to endurance exercise training. J Physiol. 2016. doi: 10. $1113 / J P 272559$

44. Scharhag-Rosenberger F, Meyer T, Walitzek S, Kindermann W. Time course of changes in endurance capacity: a 1-yr training study. Med Sci Sports Exerc. 2009:41:1130-7.

45. Ross R, de Lannoy L, Stotz PJ. Separate effects of intensity and amount of exercise on interindividual cardiorespiratory fitness response. Mayo Clin Proc. 2015;90:1506-14.

46. Dalleck LC, Van Guilder GP, Quinn EM, Bredle DL. Primary prevention of metabolic syndrome in the community using an evidence-based exercise program. Prev Med. 2013:57:392-5.

\section{Submit your next manuscript to BioMed Central and we will help you at every step:}

- We accept pre-submission inquiries

- Our selector tool helps you to find the most relevant journal

- We provide round the clock customer support

- Convenient online submission

- Thorough peer review

- Inclusion in PubMed and all major indexing services

- Maximum visibility for your research

Submit your manuscript at www.biomedcentral.com/submit
) Biomed Central 